\title{
Uptake of Single-Walled Carbon Nanotubes Conjugated with DNA by Microvascular Endothelial Cells
}

\author{
Joseph Harvey, ${ }^{1}$ Lifeng Dong, ${ }^{2}$ Kyoungtae Kim, ${ }^{3}$ Jacob Hayden, ${ }^{3}$ and Jianjie Wang ${ }^{1}$ \\ ${ }^{1}$ Department of Biomedical Sciences, Missouri State University, Springfield, MO 65897, USA \\ ${ }^{2}$ Department of Physics, Astronomy, and Materials Science, Missouri State University, Springfield, MO 65897, USA \\ ${ }^{3}$ Department of Biology, Missouri State University, Springfield, MO 65897, USA
}

Correspondence should be addressed to Jianjie Wang, jwang@missouristate.edu

Received 26 July 2011; Accepted 13 August 2011

Academic Editor: Dongwoo Khang

Copyright (c) 2012 Joseph Harvey et al. This is an open access article distributed under the Creative Commons Attribution License, which permits unrestricted use, distribution, and reproduction in any medium, provided the original work is properly cited.

\begin{abstract}
Single-walled carbon nanotubes (SWCNTs) have been proposed to have great therapeutic potential. SWCNTs conjugated with drugs or genes travel in the systemic circulation to reach target cells or tissues following extravasation from microvessels although the interaction between SWCNT conjugates and the microvascular endothelial cells (ECs) remains unknown. We hypothesized that SWCNT-DNA conjugates would be taken up by microvascular ECs and that this process would be facilitated by SWCNTs compared to facilitation by DNA alone. ECs were treated with various concentrations of SWCNT-DNA-FITC conjugates, and the uptake and intracellular distribution of these conjugates were determined by a confocal microscope imaging system followed by quantitative analysis of fluorescence intensity. The uptake of SWCNT-DNA-FITC conjugates $(2 \mu \mathrm{g} / \mathrm{mL})$ by microvascular ECs was significantly greater than that of DNA-FITC $(2 \mu \mathrm{g} / \mathrm{mL})$, observed at $6 \mathrm{hrs}$ after treatment. For the intracellular distribution, SWCNT-DNAFITC conjugates were detected in the nucleus of ECs, while DNA-FITC was restricted to the cytoplasm. The fluorescence intensity and distribution of SWCNTs were concentration and time independent. The findings demonstrate that SWCNTs facilitate DNA delivery into microvascular ECs, thus suggesting that SWCNTs serving as drug and gene vehicles have therapeutic potential.
\end{abstract}

\section{Introduction}

Emerging evidence supports the great potential of nanotechnology for medical applications with respect to diagnosis and therapeutic treatment of disease $[1,2]$. Most recently, Welsher et al. reported promising findings by using a second near-infrared window imaging system in real time [3]. They found that single-walled carbon nanotubes (SWCNTs), intravenously injected into mice, were distributed throughout the body and reached a steady level approximately $30 \mathrm{sec}$ after injection. Meanwhile, the liver, lungs, muscles, and kidneys all generated constant signal, indicating consistent blood flow in these organs. These findings indicate that SWCNTs are able to traverse the microvascular barrier into surrounding tissues. Furthermore, they reported that no toxic side effects were detected by necropsy, histology, and blood chemistry measures in those mice three months after receiving SWCNT injection [4]. The data support the promising potential of SWCNTs in medical applications, yet the mechanism(s) underlying extravasation of SWCNTs remains unknown.

Understanding how SWCNTs interact with microvascular endothelial cells (ECs) is critical for developing therapeutic treatments using highly efficient gene/drug delivery systems due to the pivotal roles of microvascular ECs in the structure and function of the microvasculature. Vascular ECs are in the vanguard for exposure to blood-born elements because they serve as the lining surface of blood vessels. In addition to being a major component of the microvascular wall, microvascular ECs dynamically mediate a variety of vascular functions, including EC-dependent vasomotion, thrombosis formation, vascular inflammation, and vascular exchange (permeability). Most drugs and genes need to be delivered to their target tissues by the systemic circulation after they are absorbed or injected into the blood stream. Subsequently, these drugs and genes traverse the vascular 
barrier and arrive at the surrounding tissues, a situation referred to as solute exchange. Importantly, all solute exchanges between circulating blood and metabolizing tissue as well as angiogenesis occur at the level of microvessels.

SWCNT uptake by microvascular ECs is an initial step in the process of drug and gene delivery into either vascular ECs or parenchymal cells. For all the therapeutic treatments with a strategy of specifically targeting microvascular ECs (e.g., antiangiogenesis to reduce tumor growth), SWCNTs conjugated with molecular cargo need to be transported into microvascular ECs. For the treatments targeting parenchymal cells (e.g., tumor cells), the SWCNT-drug conjugates must interact with microvascular ECs in the process of extravasation. In general, there are two pathways (paracellular and transcellular) responsible for large molecules to pass through the vascular barrier. Because SWCNTs often have lengths from hundreds of nanometers to several micrometers and are considered to be in the same size range as macromolecules, we predict that SWCNT-DNA conjugates will predominantly traverse the microvascular barrier via the transcellular pathway (transcytosis) [5]. Entering the microvascular ECs is an initial step in the process of transcytosis. Therefore, understanding the interaction between SWCNTs and microvascular ECs is critical for developing advanced drug and gene delivery systems using SWCNTs. To date, the effect of carbon nanotubes on microvascular ECs remains unknown.

Only a limited number of studies exist concerning the interactions between SWCNTs and vascular ECs $[6,7]$. Moreover, large vascular ECs, including HUVECs (human umbilical vein endothelial cells) or aortic ECs [8], were typically used for these studies. Heterogenic properties between large vascular and microvascular ECs $[9,10]$, however, should be considered. It is inappropriate to extrapolate the findings from studies using large vascular to microvascular ECs. Appropriately, this is the first study, to our knowledge, designed to determine the interactions between SWCNTs and microvascular ECs. We hypothesized that SWCNT-DNA conjugates would be taken up by microvascular ECs and that this process would be facilitated by SWCNTs compared with DNA alone. We employed a biocompatible SWCNTDNA conjugate $[11,12]$ labeled with FITC fluorophore (SWCNT-DNA-FITC) and primary cultured microvascular ECs derived from rat skeletal muscles [13] to study the interactions between them. We report here that SWCNT-DNAFITC conjugates were detected in the nuclei of microvascular ECs at 6 hrs after the cells were exposed to the conjugates (at a concentration as low as $2 \mu \mathrm{g} / \mathrm{mL}$ ), while DNA-FITC alone was restricted to the cytoplasm under identical conditions. Quantitative analysis of confocal images provided evidence to support the hypothesis that SWCNTs facilitated DNA uptake by microvascular ECs.

\section{Materials and Methods}

2.1. Preparation of SWCNT-DNA-FITC Conjugates. To prepare an aqueous SWCNT solution, $1 \mathrm{mg}$ of SWCNT powder (BuckyUSA Company) was dispersed in $1 \mathrm{~mL}$ ssDNA (5' CCT GAG CCA TGA TCA AAC CTG TGC AGT) or DNA-
FITC (5' FITC CCT GAG CCA TGA TCA AAC CTG TGC AGT, Alpha DNA, $1 \mathrm{mg} / \mathrm{mL}$ ) solution. The suspension was sonicated on ice for 60 minutes to avoid the degradation of DNA molecules and then centrifuged at 120,000 $\mathrm{g}$ for 60 minutes. After centrifugation, the supernatant containing individual SWCNTs was decanted, whereas the precipitates containing catalyst particles, bundled nanotubes, and amorphous carbon debris were discarded $[11,12]$.

2.2. Rat Microvascular ECs. Microvascular ECs were isolated from abdominal skeletal muscles of rats, cultured with M199 medium containing essential supplements, and characterized as previously described [13]. Briefly, the excised abdominal skeletal muscles were digested with an enzyme solution consisting of dispase $(0.12 \mathrm{mg} / \mathrm{mL}$; Worthington, Lakewood, $\mathrm{NJ})$, trypsin $(0.12 \mathrm{mg} / \mathrm{mL}$; Invitrogen, Carlsbad, Calif), collagenase type II $(0.84 \mathrm{mg} / \mathrm{mL})$, and bovine serum albumin (BSA; $1.62 \mathrm{mg} / \mathrm{mL}$ ) in M199 medium. Microvascular ECs were isolated by using Dynabeads (Dynal, Brown Deer, Wis) coated with Griffonia simplicifolia lectin and then cultured. The culture medium consisted of M199 medium supplemented with $5 \mathrm{mg} / \mathrm{mL}$ endothelial growth supplement, $0.1 \mathrm{mg} / \mathrm{mL}$ glutamine, $10000 \mathrm{unit} / \mathrm{mL}$ antibiotic/antimycotic (Gibco, Carlsbad, Calif), 5 unit/mL heparin sodium (Sigma Aldrich, St. Louis, Mo), and 20\% Fetal Bovine Serum (FBS; HyClone Lab, Inc. Logan, Utah) for the first two passages, then $10 \%$ FBS afterward.

\subsection{Nanoparticle Distribution in Primary Cultured Microvas-} cular ECs. ECs were seeded on gelatin (0.2\%)-coated LabTek II Chamber Slides with a cell density of $10^{4}$ cells $/ \mathrm{cm}^{2}$. Grown to approximately $80 \%$ confluency in $48 \mathrm{hrs}$, cells were treated with either SWCNT-DNA-FITC or DNA-FITC solution at various concentrations. After various incubation times, ECs were fixed by using $2 \%$ paraformaldehyde for $10 \mathrm{~min}$ at $37^{\circ} \mathrm{C}$ and subsequently stained with $1 \%$ DAPI $(0.5 \mu \mathrm{g} / \mathrm{mL})$ for $1 \mathrm{~min}$ at room temperature. Dulbecco's phosphate buffered saline (DPBS) containing $0.05 \%$ Tween20 (DPBST-20) was used to wash the cells following fixation and staining procedures.

2.4. Spinning Confocal Microscopy. The microvascular ECs were examined with an Olympus IX81 inverted microscope equipped with a laser light source, motorized stage capable of $1 \mu \mathrm{m} z$-axis increments, spinning confocal box (Yokogawa X1M1L), and electron multiplying EM-CCD camera (Hamamatsu, ImagEM). All the images were acquired with a 60X oil immersion objective lens and identical exposure time and appropriate filter setup controlled by Slidebook software (v5.0).

2.5. Quantification of Fluorescence Intensity. Fluorescence intensity of SWCNT-DNA-FITC or DNA-FITC in microvascular ECs was quantitatively analyzed by using Image-Pro Plus 6.0 software. Briefly, a background signal level for each image was set using the average optical density (OD) value from four regions (each region contains $>15,000$ square pixels), where no cells or any fluorescence were detected. 


\section{$2 \mu \mathrm{g} / \mathrm{mL}$ SWCNT-DNA-FITC for $24 \mathrm{hr}$}
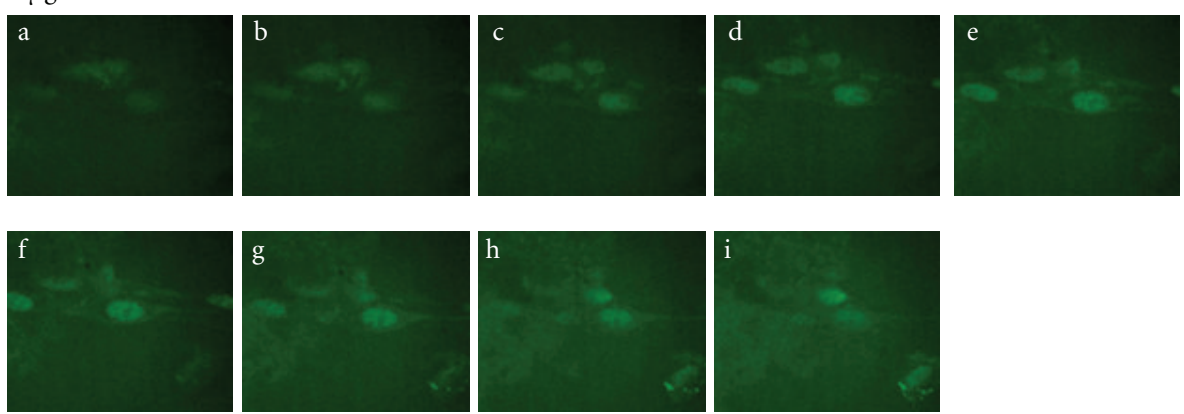

$2 \mu \mathrm{g} / \mathrm{mL}$ DNA-FITC for $24 \mathrm{hr}$
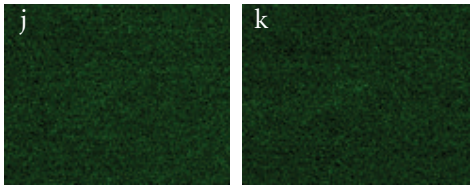

(A)
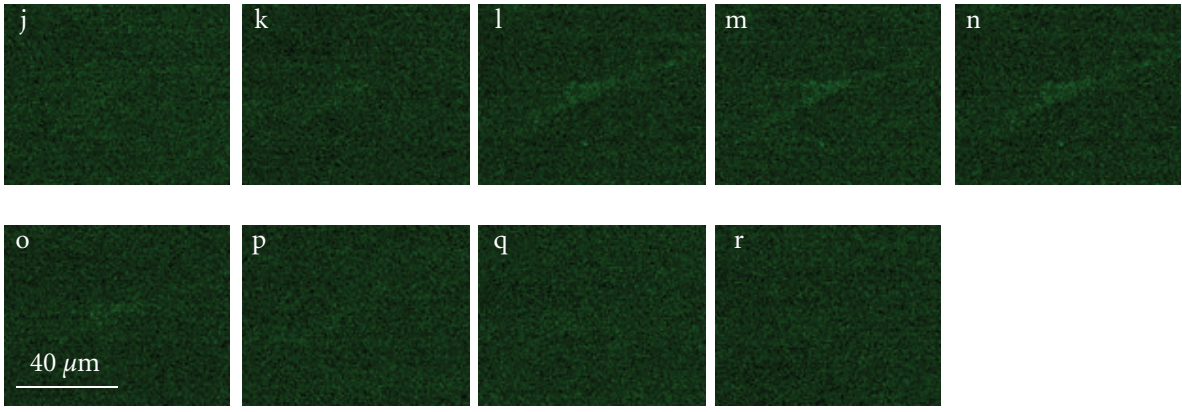

(B)

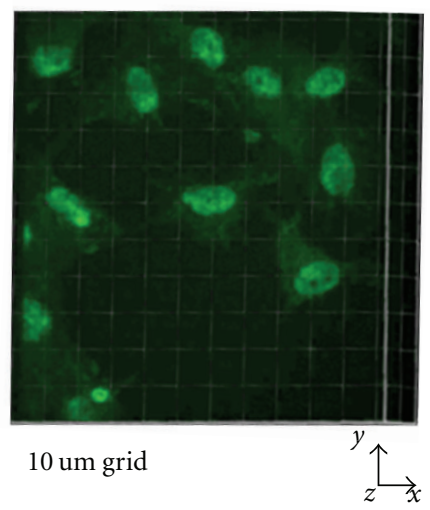

(C)

FIGURE 1: A series of z-stack images demonstrating differential distribution of SWCNT-DNA-FITC $(2 \mu \mathrm{g} / \mathrm{mL})$ versus DNA-FITC $(2 \mu \mathrm{g} / \mathrm{mL})$ conjugates in microvascular ECs: (A) (a to i), representative optical section series of SWCNT-DNA-FITC conjugates in microvascular ECs at $24 \mathrm{hrs}$ post incubation; (B) ( $\mathrm{j}$ to $\mathrm{r}$ ), representative optical section series of DNA-FITC present in microvascular ECs at 24 hrs post incubation; (C), sample 3-D image from a video (supplemental materials) acquired from ECs by a spinning confocal imaging system 48 hrs after incubation with SWCNT-DNA-FITC $(2 \mu \mathrm{g} / \mathrm{mL})$. The scale bar in (B) (o) is applicable to all images (a-r).

Consequently, each OD value obtained from this image was subtracted from the background OD. To assess fluorescence intensity of SWCNT-DNA-FITC or DNA-FITC within a cell, 5 areas of interest (AOI) from each cell were selected. The average OD value from 5 identical AOI within a cell represented the fluorescence intensity for this cell. Each image OD value, calculated as the average OD from up to 5 cells per image, represented one experiment $(n=1)$. The criteria for cell and AOI selection were as follows: (1) cells with well-defined cell shape and the strongest signal; (2) each
AOI (100 200 pixels ${ }^{2}$ in surface area) with the brightest area in the cell body excluding cell processes.

2.6. Statistical Analysis. All values are presented as mean \pm SEM. GraphPad Prism 5 software was employed for statistical analysis. Comparisons between two groups, SWCNT-DNAFITC versus DNA-FITC, at given concentrations and specific time points, were analyzed by unpaired student's $t$-test. Values of $P<0.05$ were accepted as being statistically significant. 


\section{Results and Discussion}

3.1. Uptake and Intracellular Distribution of SWCNT-DNA Conjugates in Microvascular ECs. To investigate the interactions between microvascular ECs and SWCNTs, we isolated, cultured, and characterized primary microvascular ECs from rat skeletal muscles. Importantly, the results derived from this in vitro study using primary cultured microvascular ECs will guide us to investigate further the efficacy of the drug- or gene-SWCNT delivery system in species- and tissue-matched intact microvessels in living animals $[13,14]$.

Individual short SWCNTs with $800-900 \mathrm{~nm}$ in average length $[11,12]$ were used in the SWCNT-DNA-FITC conjugates. To identify the effect of SWCNTs on DNA uptake by microvascular ECs, the cells were treated with SWCNTDNA-FITC $(2 \mu \mathrm{g} / \mathrm{mL})$ and DNA-FITC $(2 \mu \mathrm{g} / \mathrm{mL})$, respectively. The images of microvascular ECs exposed to SWCNTDNA-FITC conjugates for $24 \mathrm{hrs}$ were acquired by a spinning scanning confocal imaging system and demonstrated in Figure 1. SWCNT-DNA-FITC conjugates appeared to be localized in the EC nuclei, a conclusion determined by the position, shape, and area of FITC fluorescence as shown in a set of z-stack images in Figure 1(A). In contrast, DNAFITC was dispersed weakly in the cytoplasm of microvascular ECs (Figure 1(B)) and often found on or close to the plasma membrane (Figure 2(a)), but not in the nucleus. The nuclear localization of SWCNTs was also manifested in a $3 \mathrm{D}$ projection image (Figure $1(\mathrm{C})$ ) and a supplementary z-scan video (see Supplementary Materials available online at doi: 10.1155/2012.196189). The results support our hypothesis that DNA conjugated to SWCNTs is taken up by microvascular ECs. In view of the finding that SWCNT-DNA-FITC, but not DNA-FITC, accumulates in the nucleus, it is likely that SWCNTs play a facilitating role in delivering the DNA to the nuclei of microvascular ECs. Thus, our results suggest that SWCNTs could be used as vectors for gene delivery to microvascular EC targets.

SWCNTs used in this study did not appear to have adverse effects on cellular morphology and proliferation, observations supported by the previous studies using astrocytoma cells $[11,12]$. However, the formation of aggregates was observed in the culture medium following the addition of SWCNT-DNA-FITC solution for the cell treatment groups, but not DNA-FITC solution for the control groups. The aggregates adhered to the surfaces of microvascular ECs even after fixation and washing procedures. The morphology and numbers of microvascular ECs treated with SWCNTDNA-FITC conjugates at various concentrations within 48 hrs did not differ with control ECs treated with DNAFITC alone (data not shown), assessed by a transmitted light imaging system. The functional and molecular influence of the aggregations on microvascular ECs remains to be studied.

\subsection{Quantitative Evidence for the Facilitation of DNA Uptake} by Microvascular ECs through SWCNTs. To quantitatively assess the amount of DNA conjugated to SWCNTs taken up by microvascular ECs, we analyzed the fluorescence intensity of SWCNT-DNA-FITC conjugates by measuring OD values. For this purpose, images of microvascular ECs, treated with
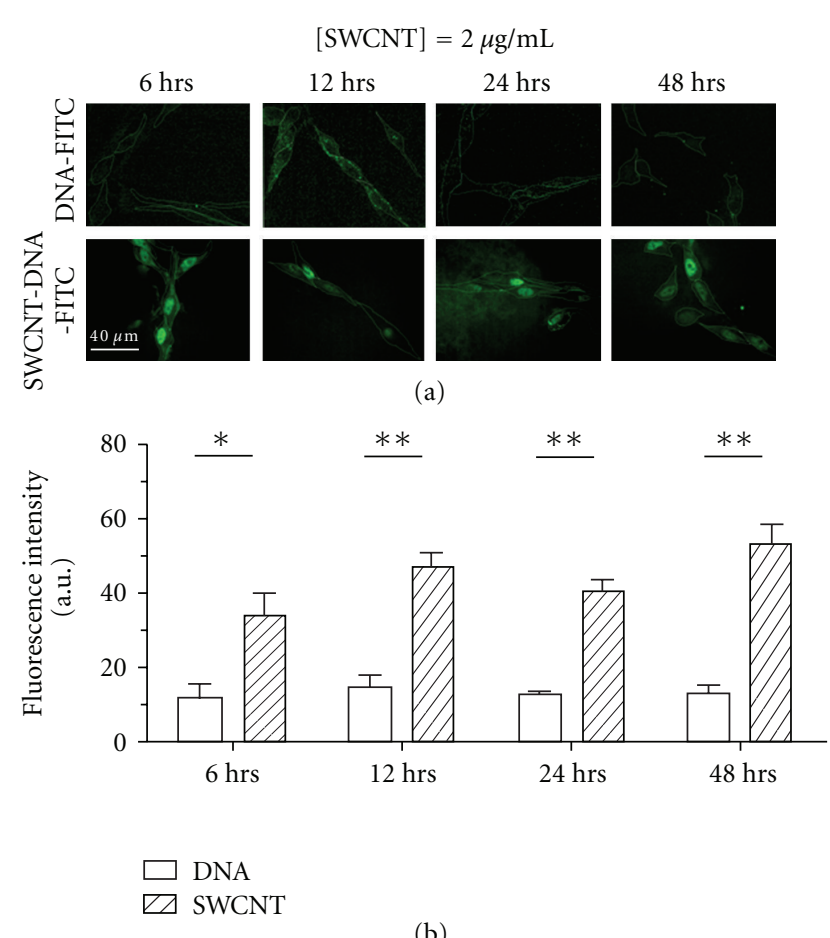

FIGURE 2: Distribution and fluorescence intensity of SWCNT-DNAFITC $(2 \mu \mathrm{g} / \mathrm{mL})$ and DNA-FITC $(2 \mu \mathrm{g} / \mathrm{mL})$ at various time intervals within $48 \mathrm{hrs}$. (a) Representative projection images suggest that SWCNT-DNA-FITC conjugates are distributed in microvascular EC nuclei (lower panel); DNA-FITC appears to be restricted to the cytoplasm (upper panel). (b) SWCNT-facilitated DNA transport into microvascular ECs. Fluorescence intensity was quantitatively assessed by measuring optical density of confocal images as described in Materials and Methods. The $P$-values less than 0.05 and 0.01 are expressed as $*^{*}$ and ${ }^{* *}$, respectively $(n=3-11)$. The scale bar is applicable to all images.

SWCNT-DNA-FITC conjugates or DNA-FITC at various concentrations $(2,4$, and $10 \mu \mathrm{g} / \mathrm{mL}$ ) for up to $48 \mathrm{hrs}$ (at $6,12,24$, and $48 \mathrm{hrs}$ ), were acquired. Interestingly, the OD value of SWCNT-DNA-FITC conjugate in microvascular ECs registered approximately a 3 4-fold increase compared to that of DNA-FITC after incubation at a concentration of $2 \mu \mathrm{g} / \mathrm{mL}(33.5 \pm 6.3(n=4)$ versus $11.3 \pm 3.8(n=3)$ at $6 \mathrm{hrs}$; $47.0 \pm 3.4(n=6)$ versus $14.8 \pm 3.4(n=6)$ at $12 \mathrm{hrs} ; 40.4 \pm 3.3$ $(n=7)$ versus $12.7 \pm 1.0(n=6)$ at $24 \mathrm{hrs} ; 53.7 \pm 5.4(n=6)$ versus $13.2 \pm 2.2(n=11)$ at $48 \mathrm{hrs})$ as shown in Figure 2. As microvascular ECs were treated with SWCNT-DNA-FITC conjugates at a concentration of $10 \mu \mathrm{g} / \mathrm{mL}$, the uptake of SWCNT-DNA-FITC by microvascular ECs was also found to be greater than that of DNA-FITC $(40.3 \pm 5.5(n=4)$ versus $12.7 \pm 2.2(n=3) 6$ hrs; $46.7 \pm 2.7(n=3)$ versus $2.3 \pm 0.9$ $(n=3)$ at $12 \mathrm{hrs} ; 38.7 \pm 7.6(n=6)$ versus $18.3 \pm 2.9(n=6)$ at $24 \mathrm{hrs} ; 37.6 \pm 4.5(n=12)$ versus $16.5 \pm 1.8(n=6)$ at $48 \mathrm{hrs})$ (Figure 3 ). These data demonstrate again that SWCNTs facilitate DNA uptake by microvascular ECs. However, this action was neither concentration dependent within the tested range from $2 \mu \mathrm{g} / \mathrm{mL}$ to $10 \mu \mathrm{g} / \mathrm{mL}$ (Figure $4(\mathrm{a})$ ) nor time dependent within the periods from $6 \mathrm{hrs}$ to $48 \mathrm{hrs}$ 


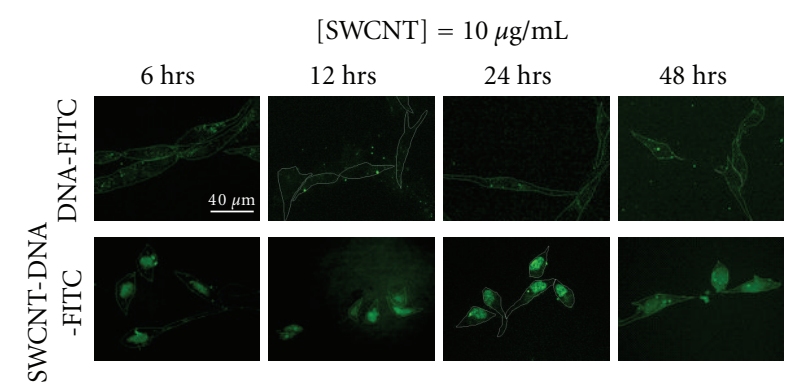

(a)

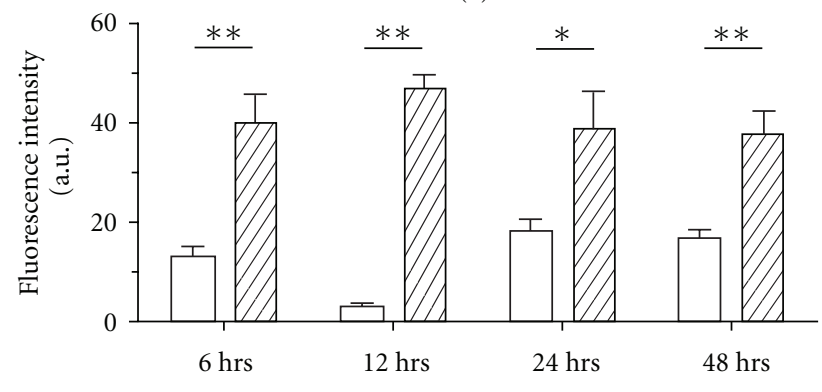

$\square$ DNA

$\square]$ SWCNT

(b)

FIGURE 3: Intracellular compartmentalization and fluorescence intensity of SWCNT-DNA-FITC $(10 \mu \mathrm{g} / \mathrm{mL})$ and DNA-FITC $(10 \mu \mathrm{g} / \mathrm{mL})$ in microvascular ECs and observed at $6,12,24$, and 48 hrs. (a) Representative projection images acquired by confocal microscopy; (b) Quantitative analysis of fluorescence intensity of SWCNT-DNA-FITC and DNA-FITC conjugates. The $P$-values less than 0.05 and 0.01 are expressed as ${ }^{*}$ and ${ }^{* *}$, respectively $(n=3-$ 12). The scale bar is applicable to all images.

(Figure 4(b)). Although there were no major differences in the fluorescence intensity of internalized SWCNT-DNAFITC conjugates over the time course and among treatments with various concentrations of SWCNTs, we observed a trend that $2 \mu \mathrm{g} / \mathrm{mL}$ SWCNTs combined with $12 \mathrm{hrs}$ incubation yields optimal, efficacious delivery (Figure 4). Therefore, for future studies, we recommend that microvascular ECs be treated with $2 \mu \mathrm{g} / \mathrm{mL}$ SWCNT-DNA-FITC for $12 \mathrm{hrs}$ in order to understand mechanisms by which SWCNTs enter microvascular ECs.

The mechanisms underlying SWCNT uptake by microvascular ECs remain unknown at the present time. In general, there are three ways by which extracellular molecules enter a cell: simple diffusion, carrier-mediated transport (e.g., ion channels or transporters), and endocytosis. SWCNTs, macromolecules, are internalized into the intracellular compartment through endocytosis, evidenced by several studies using different types of mammalian cells [6, 15]. Endocytosis can be further categorized as pinocytosis, phagocytosis, and clathrin- and caveolae-mediated endocytosis. Pinocytosis and phagocytosis are main pathways for macrophages, while clathrin- and caveolae-mediated pathways are used by most cells including endothelial cells [16, 17]. Kam found that the uptake of SWCNT-DNA conjugates by two tumor cell lines, HeLa and HL60 tumor cells, was attenuated by disrupting the formation of clathrincoated vesicles. The evidence supports the internalization of SWCNT-DNA via clathrin-mediated endocytotic mechanism [15]. Compared with Kam's study, the current one used primary skeletal muscle microvascular ECs derived from the exchange microvessels to investigate the uptake of SWCNT-DNA. To our best knowledge, different types of cells have heterogeneous functions, structures, and mechanisms, even within vascular endothelial cells across vascular trees [10]. Lining the inner side of exchange microvessels, microvascular ECs used in the current study engage in transcytosis to allow macromolecules traversing the microvascular barrier. Endothelial cells possess more caveolae than clathrin-coated pits. The number of caveolae is the highest in capillary endothelial cells, compared with arteries, arterioles, venules, and veins, particularly in heart, lung, and skeletal muscle [10]. In the process of transcytosis, caveolae-mediated endocytosis plays an important role as an initial step of the transcellular pathway across endothelial cells $[5,10,18]$. In addition to clathrinand caveolae-mediated endocytosis, Muro and coworkers demonstrate that nanoparticles, composed of polystyrene latex microspheres, were taken up by HUVECs via the celladhesion-molecule- (CAM-) mediated endocytotic process. The CAM-mediated endocytosis was revealed by colocalization of nanoparticles and endocytotic markers, including early endosome antigen [19] and $\mathrm{Na}+\mathrm{H}+$ exchange ion channel (associated with CAM-mediated endocytosis) [20]. It was noted that Muro's study used HUVEC, while we used microvascular EC besides different nanoparticles. In addition, studies using a mathematical model to calculate the energy cost needed for carbon nanotubes piercing through the bilayer of the plasma membrane demonstrate indirectly that the most likely pathway of carbon nanotube delivery into cells is by endocytosis due to a substantial energy requirement [21].

So far, the answer to whether or not microvascular ECs take up SWCNTs by endocytotic processes and what endocytotic mechanisms are employed remain to be determined. Understanding these mechanisms requires characterizing the spatial-temporal relationship between SWCNTs and microvascular ECs.

We found that the fluorescence intensity of the SWCNTDNA-FITC conjugate in microvascular ECs was concentration and time independent. These results indicate that SWCNTs do not accumulate over time in microvascular ECs and that the level of SWCNTs in cells reached a plateau $6 \mathrm{hrs}$ after incubation. Since SWCNTs are highly stable structures [6], we predict that microvascular ECs are not able to degrade internalized SWCNTs via lysosomes but expel them over time (exocytosis). The process, in which molecules, in this case of SWCNTs, undergo endocytosis and then exocytosis, is referred to as transcytosis. Transcytosis is one of the pathways by which macromolecules, such as drugs and large proteins, pass through microvascular barriers $[5,22]$. This concept has significant implications in terms of using SWCNTs as carriers to deliver drugs/genes into parenchymal cells after SWCNTs move from microvessels 


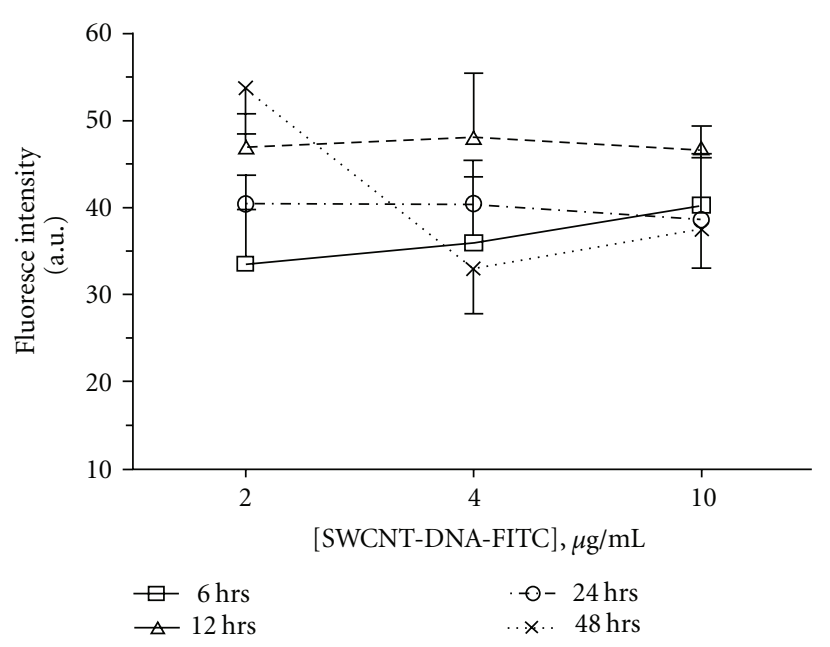

(a)

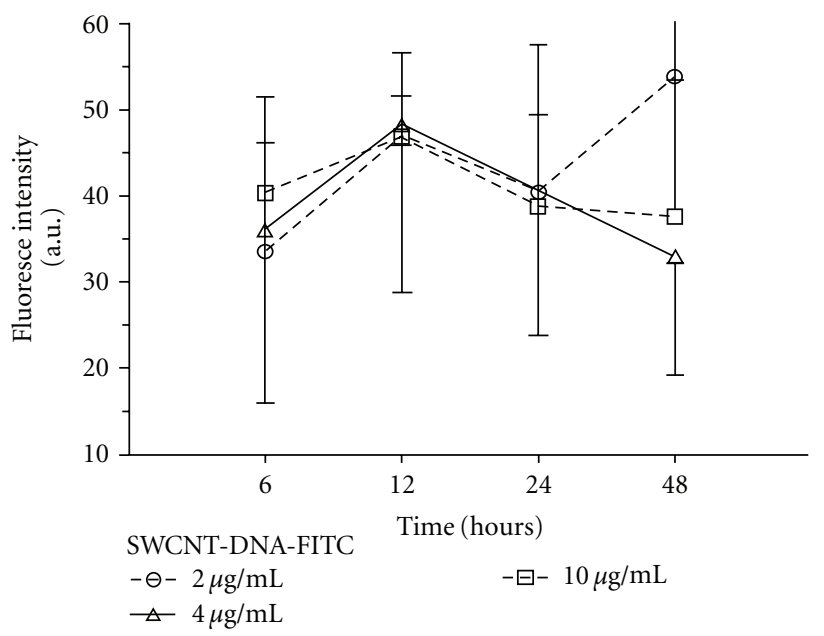

(b)

FIGURE 4: Quantity of SWCNT-DNA-FITC conjugates retained in EC nuclei is concentration- and time-independent. (a) Relationship between quantity of SWCNTDNA-FITC in the nuclei and the concentrations of SWCNT-DNA-FITC conjugates; (b) Time-course of fluorescence intensity of SWCNT-DNA-FITC in the nuclei of microvascular ECs.

into the surrounding interstitium. However, the timing of DNA release from SWCNT-DNA conjugates, critical information for designing a drug delivery system with high efficacy, remains to be studied.

\section{Conclusions}

This study is the first to demonstrate that SWCNT-DNA conjugates are taken up by microvascular ECs. We observed that intracellular fluorescence intensity of SWCNT-DNAFITC in microvascular ECs was greater than the fluorescence intensity of DNA-FITC alone, suggesting that the uptake was facilitated by SWCNTs. In addition, SWCNT-DNA conjugates were most likely localized in the nuclei of microvascular ECs, whereas DNA was distributed in the cytoplasm. The
SWCNT uptake by microvascular ECs was concentration and time independent. These data suggest that SWCNTs may provide impressive therapeutic potential as vehicles for delivery of drugs or genes to either microvascular ECs or parenchymal cells.

\section{Acknowledgments}

The authors thank Missouri State University and the Cottrell College Science Award from the Research Corporation for Science Advancement for supporting this work. The spinning confocal microscope imaging system was supported by the National Science Foundation (Award no. MRI-RUI 0923024). They gratefully thank Dr. Michael Craig for critical suggestions and discussions about the paper.

\section{References}

[1] F. Liang and B. Chen, "A review on biomedical applications of single-walled carbon nanotubes," Current Medicinal Chemistry, vol. 17, no. 1, pp. 10-24, 2010.

[2] Z. Liu, K. Chen, C. Davis et al., "Drug delivery with carbon nanotubes for in vivo cancer treatment," Cancer Research, vol. 68, no. 16, pp. 6652-6660, 2008.

[3] K. Welsher, S. P. Sherlock, and H. Dai, "Deep-tissue anatomical imaging of mice using carbon nanotube fluorophores in the second near-infrared window," Proceedings of the National Academy of Sciences of the United States of America, vol. 108, no. 22, pp. 8943-8948, 2011.

[4] Z. Liu, C. Davis, W. Cai, L. He, X. Chen, and H. Dai, "Circulation and long-term fate of functionalized, biocompatible single-walled carbon nanotubes in mice probed by Raman spectroscopy," Proceedings of the National Academy of Sciences of the United States of America, vol. 105, no. 5, pp. 1410-1415, 2008.

[5] D. Mehta and A. B. Malik, "Signaling mechanisms regulating endothelial permeability," Physiological Reviews, vol. 86, no. 1, pp. 279-367, 2006.

[6] A. Albini, V. Mussi, A. Parodi et al., "Interactions of singlewall carbon nanotubes with endothelial cells," Nanomedicine, vol. 6, no. 2, pp. 277-288, 2010.

[7] W. W. Cheng, Z. Q. Lin, B. F. Wei et al., "Single-walled carbon nanotube induction of rat aortic endothelial cell apoptosis: reactive oxygen species are involved in the mitochondrial pathway," International Journal of Biochemistry and Cell Biology, vol. 43, no. 4, pp. 564-572, 2011.

[8] L. Zhiqing, X. Zhuge, C. Fuhuan et al., "ICAM-1 and VCAM-1 expression in rat aortic endothelial cells after singlewalled carbon nanotube exposure," Journal of Nanoscience and Nanotechnology, vol. 10, no. 12, pp. 8562-8574, 2010.

[9] W. C. Aird, "Phenotypic heterogeneity of the endothelium: II. Representative vascular beds," Circulation Research, vol. 100, no. 2, pp. 174-190, 2007.

[10] W. C. Aird, "Phenotypic heterogeneity of the endothelium: I. Structure, function, and mechanisms," Circulation Research, vol. 100, no. 2, pp. 158-173, 2007.

[11] L. Dong, K. L. Joseph, C. M. Witkowski, and M. M. Craig, "Cytotoxicity of single-walled carbon nanotubes suspended in various surfactants," Nanotechnology, vol. 19, no. 25, Article ID 255702, 2008.

[12] L. Dong, C. M. Witkowski, M. M. Craig, M. M. Greenwade, and K. L. Joseph, "Cytotoxicity effects of different surfactant 
molecules conjugated to carbon nanotubes on human astrocytoma cells," Nanoscale Research Letters, vol. 4, no. 12, pp. 1517-1523, 2009.

[13] J. Wang, S. Bingaman, and V. H. Huxley, "Intrinsic sexspecific differences in microvascular endothelial cell phosphodiesterases," American Journal of Physiology-Heart and Circulatory Physiology, vol. 298, no. 4, pp. H1146-H1154, 2010.

[14] J. Wang and V. H. Huxley, "Adenosine A2A receptor modulation of juvenile female rat skeletal muscle microvessel permeability," American Journal of Physiology-Heart and Circulatory Physiology, vol. 291, no. 6, pp. H3094-H3105, 2006.

[15] N. W. S. Kam, Z. Liu, and H. Dai, "Carbon nanotubes as intracellular transporters for proteins and DNA: an investigation of the uptake mechanism and pathway," Angewandte ChemieInternational Edition, vol. 45, no. 4, pp. 577-581, 2006.

[16] R. V. Stan, "Endocytosis pathways in endothelium: how many?" American Journal of Physiology-Lung Cellular and Molecular Physiology, vol. 290, no. 5, pp. L806-L808, 2006.

[17] B. S. Ding, T. Dziubla, V. V. Shuvaev, S. Muro, and V. R. Muzykantov, "Advanced drug delivery systems that target the vascular endothelium," Molecular Interventions, vol. 6, no. 2, pp. 98-112, 2006.

[18] P. Oh, P. Borgström, H. Witkiewicz et al., "Live dynamic imaging of caveolae pumping targeted antibody rapidly and specifically across endothelium in the lung," Nature Biotechnology, vol. 25, no. 3, pp. 327-337, 2007.

[19] S. Muro, X. Cui, C. Gajewski, J. C. Murciano, V. R. Muzykantov, and M. Koval, "Slow intracellular trafficking of catalase nanoparticles targeted to ICAM-1 protects endothelial cells from oxidative stress," American Journal of PhysiologyCell Physiology, vol. 285, no. 5, pp. C1339-C1347, 2003.

[20] S. Muro, M. Mateescu, C. Gajewski, M. Robinson, V. R. Muzykantov, and M. Koval, "Control of intracellular trafficking of ICAM-1-targeted nanocarriers by endothelial $\mathrm{Na}+$ / $\mathrm{H}+$ exchanger proteins," American Journal of PhysiologyLung Cellular and Molecular Physiology, vol. 290, no. 5, pp. L809-L817, 2006.

[21] S. Pogodin and V. A. Baulin, "Can a carbon nanotube pierce through a phospholipid bilayer?" ACS Nano, vol. 4, no. 9, pp. 5293-5300, 2010.

[22] C. C. Michel and F. E. Curry, "Microvascular permeability," Physiological Reviews, vol. 79, no. 3, pp. 703-761, 1999. 

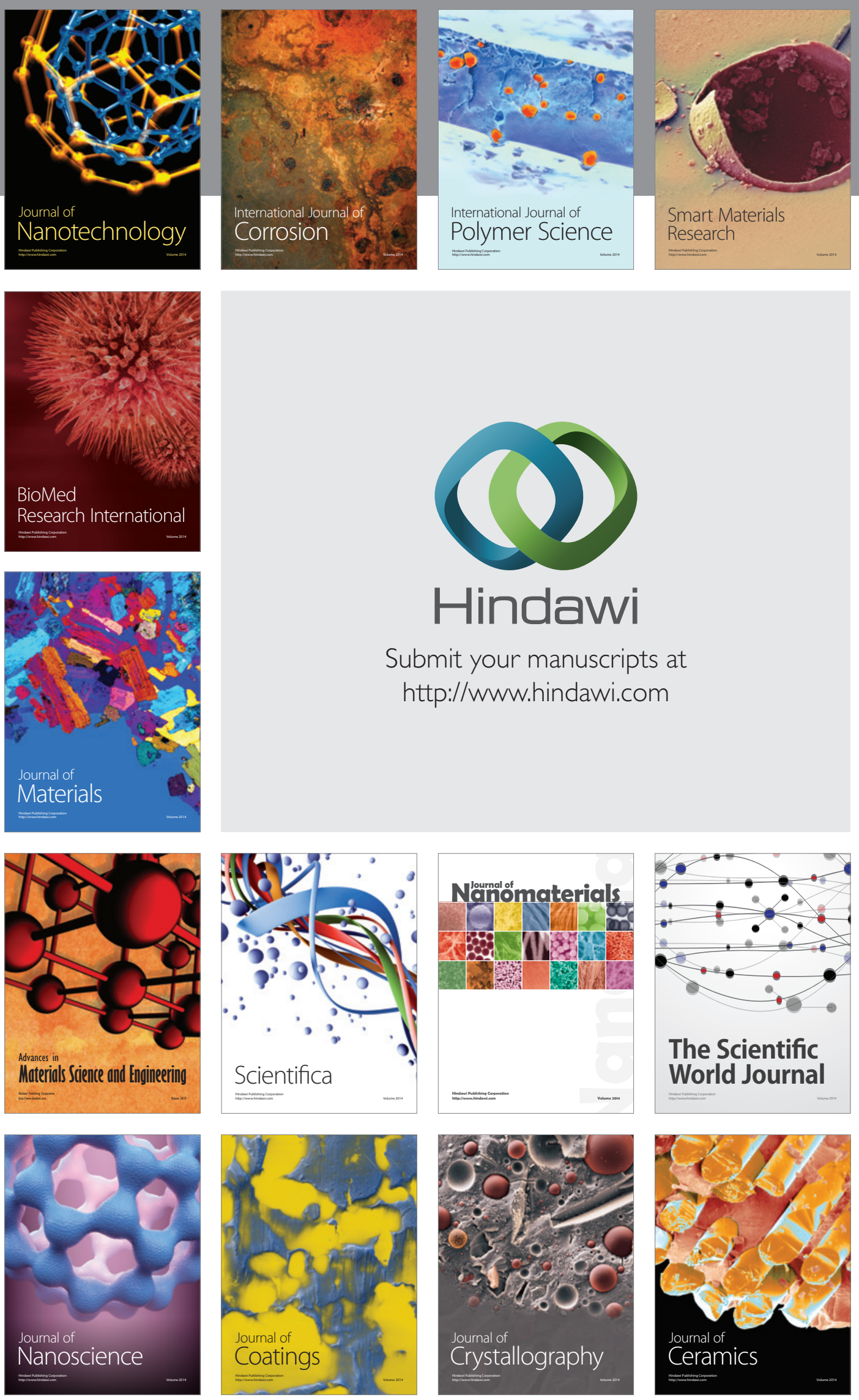

The Scientific World Journal

Submit your manuscripts at

http://www.hindawi.com

\section{World Journal}

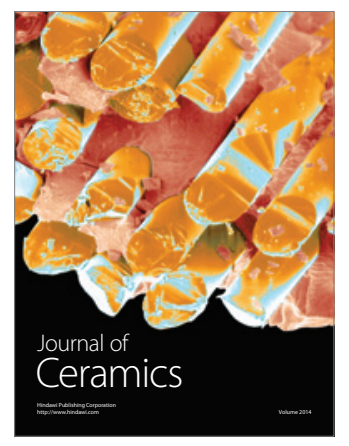

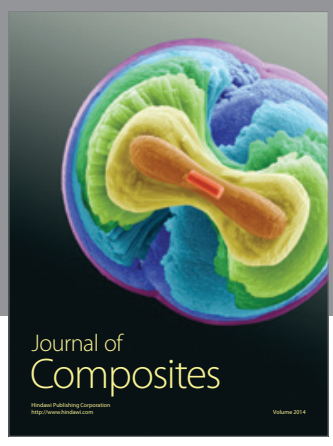
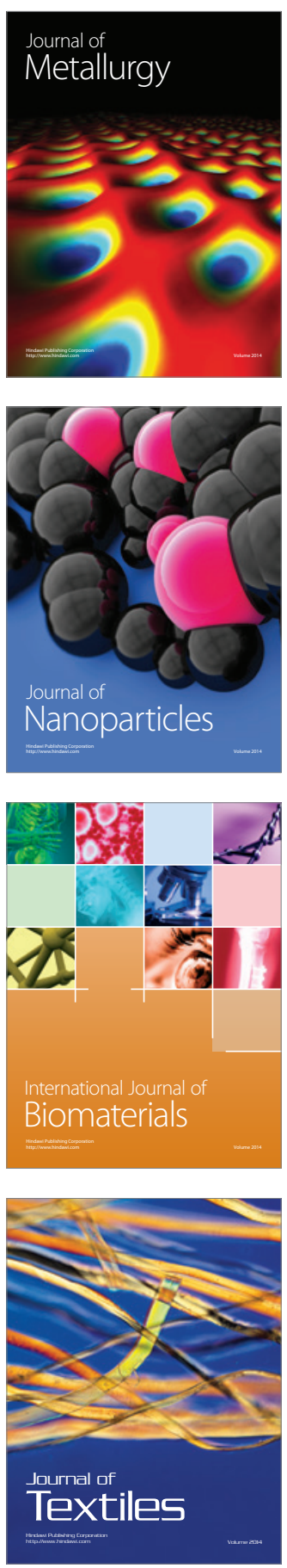